\title{
CLOSED KINETIC CHAIN EXERCISES FOR TRAINING OF THE DYNAMIC STABILIZATION IN SHOULDER IMPINGEMENT SYNDROME
}

\author{
Lyubomira Sazdova
}

\begin{abstract}
Introduction. In modern literature impingement syndrome of the shoulder is defined as a group of symptoms, rather than a specific diagnosis. A number of underlying pathologies can lead to symptoms of impingement, including: muscle imbalance or dysfunction, rotator cuff pathologies, glenohumeral instability, or scapular dyskinesis. If left untreated or misdiagnosed, partial-thickness and full-thickness rotator cuff tears may result.

The aim of this report is to present closed kinetic chain exercises for training of the dynamic stabilization of the shoulder and to evaluate the effect of their application in patients with secondary impingement syndrome.

Material and Methods. The study was conducted on 14 subjects - 9 men and 5 women, mean age 28.7, all with shoulder impingement or compressive symptoms. The specialized physiotherapeutic program included closed kinetic chain exercises to address scapular dyskinesis and rotator cuff dysfunction. To evaluate the functional condition and response to the treatment were examined: pain intensity, painless active range of motion, and scapular stability and symmetry.

Results. The results of application of the specialized therapeutic exercises showed statistically significant decrease in pain level, increased pain-free range of motion and improved scapular stability.

Conclusion. Physiotherapy in shoulder impingement syndrome is a complex process that requires thorough analysis and detailed clinical examination to determine the causes of this problem. Once muscle strength and balance are restored, functional kinetic chain exercises and patterns of muscle activation should be incorporated throughout the physiotherapy process.
\end{abstract}

Key words: shoulder impingement, closed-chain exercises, physiotherapy.

Introduction. In modern literature impingement muscle imbalance.

syndrome of the shoulder is defined as a group of symptoms, rather than a specific diagnosis. A wide range of different factors and underlying pathologies can lead to symptoms of impingement, including: muscle imbalance or dysfunction, rotator cuff pathologies, glenohumeral instability, or scapular dyskinesis. If left untreated or misdiagnosed, partial-thickness and full-thickness rotator cuff tears may result.

In secondary shoulder impingement syndrome (SIS) stability of the humeral head is often compromised. A major role for the stabilization of the head of the humerus plays the muscles of the rotator cuff (Supraspinatus, Infraspinatus, Teres minor, Subscapularis). In cases of impingement commonly humeral head translates superiorly, subacromial space decreases and soft tissues get pinched. This glenohumeral instability may be due to force couple dysfunction (deltoid - rotator cuff), delayed muscle activation, decreased muscle strength or

On the other hand, scapular position and movement are also critical components of normal glenohumeral function. There are evidences, suggesting that in SIS kinematic of scapulothoracic motions is impaired $(9,4,5)$, condition also known as scapular dyskinesis. Stability of the scapula depends on the surrounding musculature, which must dynamically position the glenoid, so that efficient glenohumeral movement can occur. The main scapula stabilizers are Levator Scapulae, Rhomboids, Serratus Anterior, and Trapezius. When weakness or dysfunction is present in these muscles, normal scapular positioning and mechanics may become altered, which result in abnormal stress to the capsular structures, rotator cuff compression and impaired performance (14). Changes in resting position of the scapula, often seen in patients with SIS, can be presented as increased anterior tilting and lack of posterior tilting, increased internal rotation of the scapula and decreased upward rotation, or scapu- 
lar winging $(7,9,10,11)$. Problems associated with changes in scapular kinematics during dynamic arm movements are: altered muscle activity patterns in the scapular muscles (9), decreased muscular activity or strength, or change in the timing properties of the Serratus Anterior, the Lower Trapezius and Middle Trapezius, and increased activity in the upper part of the Trapezius $(2,9)$.

In conservative approach in the treatment of SIS, exercise therapy has an important role. New insights emphasize that the dynamic stabilization of the shoulder and scapula is an essential part of the management (1). Unfortunately, the scapular musculature is often neglected in the treatment of SIS. This lack of attention may often lead into the incomplete treatment (14). Therefore, physiotherapy for overhead athletes with symptoms of impingement and scapular dyskinesis has to be addressed towards reestablishment of normal shoulder function by restoration of dynamic stability, muscular balance, endurance and proprioception and neuromuscular control.

The purpose of this study is to present closed kinetic chain exercises for training of the dynamic stabilization of scapula and shoulder, and to evaluate the effect of their application in patients with secondary shoulder impingement syndrome.

Material and Methods. Contingent of the study. The study was conducted on 14 subjects - 9 men and 5 women, mean age 28.7 , with unilateral shoulder pain (the dominant upper limb was affected) ( $\mathrm{Ta}$ ble 1). All participants are active athletes (playing volleyball, tennis and beach tennis) with shoulder impingement or compressive symptoms, with no history for operative treatment.

Table 1. Contingent of the study.

\begin{tabular}{|c|c|c|c|c|c|}
\hline \multirow{2}{*}{$\begin{array}{c}\text { Contingent of } \\
\text { the study }\end{array}$} & \multicolumn{2}{|c|}{ Sex } & $\begin{array}{c}\text { Average } \\
\text { age }\end{array}$ & \multicolumn{2}{c|}{ Affected side } \\
\cline { 2 - 3 } & Male & Female & \multirow{2}{*}{28.7} & Left & Right \\
\cline { 2 - 3 } \cline { 5 - 6 } & 9 & 5 & & 3 & 11 \\
\hline
\end{tabular}

Methods for functional assessment. To evaluate the functional condition and response to the treatment were examined: (1) pain intensity by visual analogue scale (VAS); (2) pain-free active shoulder range of motion (ROM) by a standard goniometer; (3) scapular stability and symmetry - scapular tilt, rotation, winging, measurement of the distance between the inferior angle of the scapula and the adja- cent spinous process (in resting position and in $90^{\circ}$ shoulder abduction). All measurements were taken before and after 8 -week treatment period.

The specialized physiotherapeutic program included closed kinetic chain exercises to restore muscle activation patterns and improve the dynamic stabilization of the shoulder and scapula. The level of difficulty of the exercises increased based on the quality of the performance and perceived intensity of pain.

For patients with SIS with presumed weakness of the Serratus Anterior, activation of this muscle is of particular importance. One of the best exercises for training of the Serratus Anterior is push-up plus, where the plus phase refers to the posterior translation of the thorax on a fixed scapula, resulting in scapular protraction. In this exercise Serratus Anterior shows increased activity with decreased activity of the Upper Trapezius (8). In our therapeutic program this exercise started from standing position (wall push-up plus), and later was performed from standard (Fig.1), and plank position - Plank push-up plus (Fig.2).Another exercise for activating the Serratus Anterior is wall slides. According some research, the amount of Serratus Anterior activation during this exercise is not significantly different than the amount of Serratus Anterior activation during the plus phase of the wall push-up plus exercise (3). The wall slide exercise, however, allows for this activation overhead (above $90^{\circ}$ of shoulder elevation), where patients with shoulder impingement have been shown to have altered scapular mechanics and decreased Serratus Anterior activity. For our therapeutic program we choose wall slides to be performed with foam roller against the wall (Fig.3). The level of difficulty of this exercise was increased by the use of the Theraband loop (Fig.4). During the wall slides with Theraband loop additional tension of the external rotators of the shoulder was added, which elicits depression of the humeral head and decreases Pectoralis Major activity. The level of the resistance was adjusted accordingly for all subjects throughout the treatment process. To increase the difficulty of this exercise and resistance over the foam roller patients step back from the wall. The progression of wall slides was by using a stability ball.Another exercise for strengthening of the posterior chain of the shoulder and training of the rotator cuff and scapular stabilization is scapular-clock exercise - small ball circles against the 
wall (clockwise and counter clockwise) (Fig. 5, 6). It develops proprioception, balance and coordination of the shoulder and scapular muscles. The exercise was performed in different starting positions of the shoulder elevation $\left(90^{\circ}, 120^{\circ}\right)$, and with different patient's positions (facing the wall or sideways the wall).

Another exercise for strengthening of the posterior chain of the shoulder and training of the rotator cuff and scapular stabilization is scapular-clock exercise - small ball circles against the wall (clockwise and counter clockwise) (Fig. 5, 6). It develops proprioception, balance and coordination of the shoulder and scapular muscles. The exercise was performed in different starting positions of the shoulder elevation $\left(90^{\circ}, 120^{\circ}\right)$, and with different patient's positions (facing the wall or sideways the wall).

When the quality of the performance improved progression in the therapeutic program was made by including exercises from quadruped and plank position. Exercises for training of the dynamic sta-

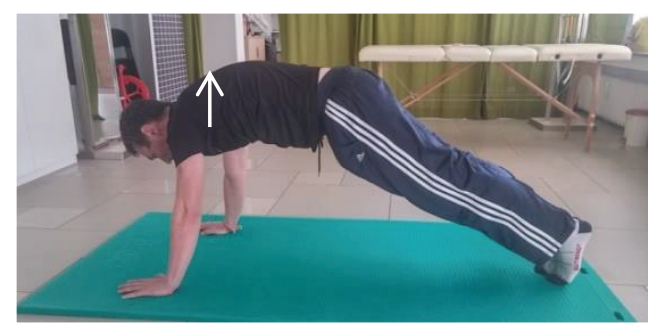

Fig.1. Push-up plus.

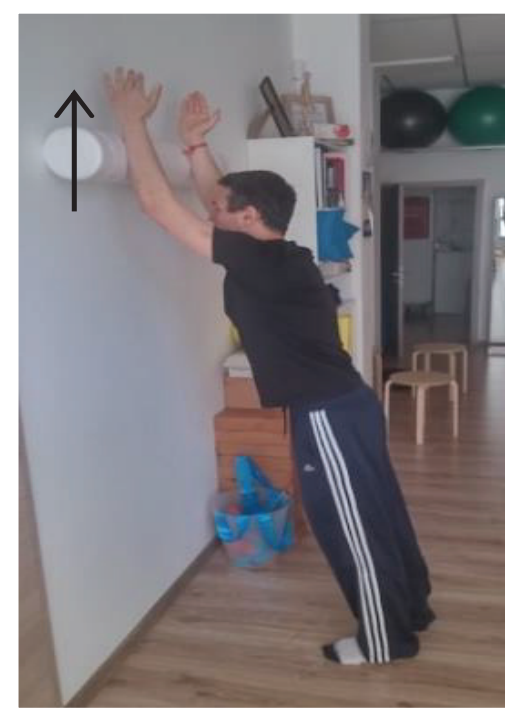

Fig.3. Wall slides with foam roller. bilization from these positions are connected with heavy loading, so it is important patients to keep good scapular control - no winging or excessive protraction or rotation of the scapula. Because of the easier scapular control, at the beginning the following exercises were performed from quadruped position, then from plank position.

Exercises started with plank body saw - moving the body forward/ backward (Fig.7). For training of scapular stabilizers and rotator stability of the shoulder were included plank rotations (Fig. 8) and archer turns (Fig. 9) - rotation of the body from plank position and with extended elbow.

For multidirectional training of the dynamic stabilization patients performed quadruped 4-way reaching - reaching forward/ backward/ left/ right form quadruped position (Fig. 10), and quadruped lateral/ forward/ backward crawls. Progression in these exercises was made by using Theraband loop (Fig. 11).

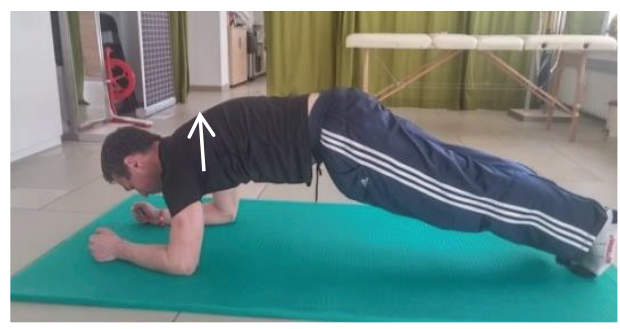

Fig. 2. Plank Push-up plus.

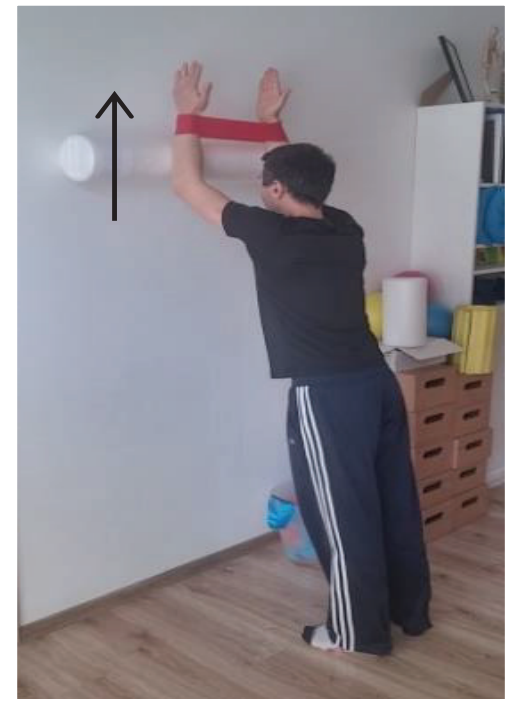

Fig.4 Wall slides with Theraband loop. 


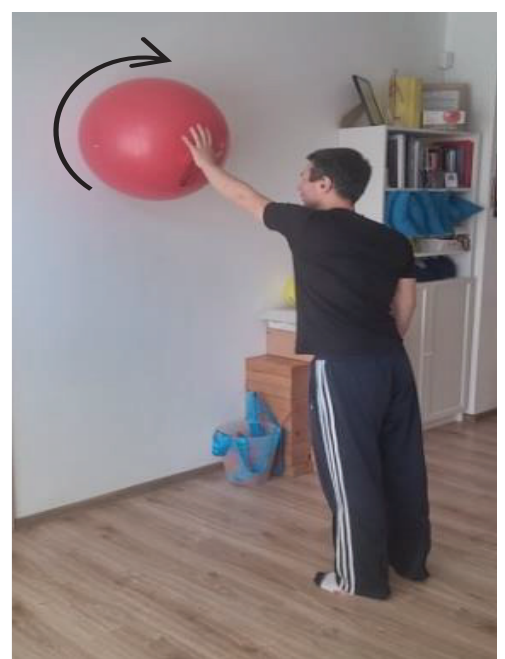

Fig. 5. Clock exercise with therapeutic ball.

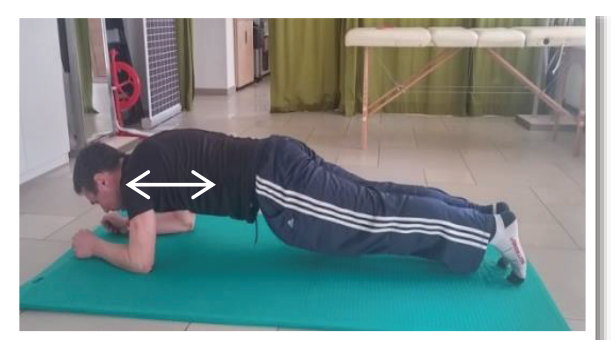

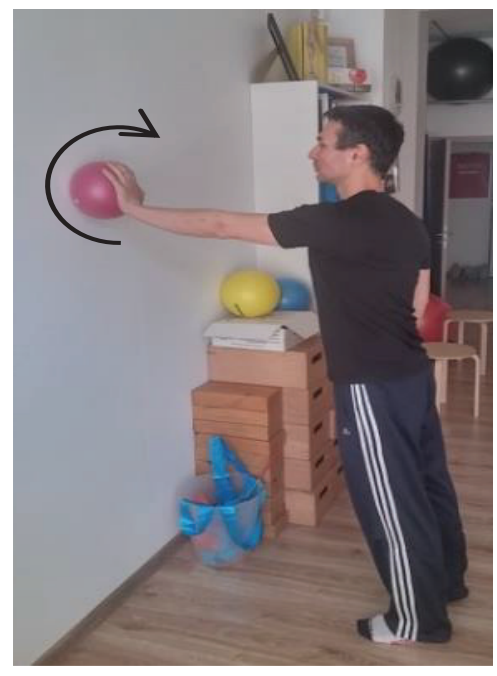

Fig. 6. Clock exercise with small ball.

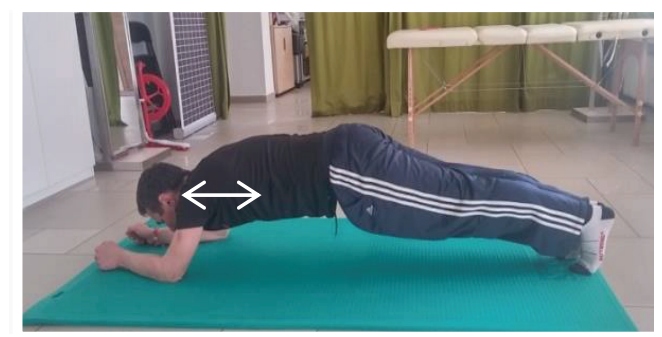

Fig.7. Plank body saw.

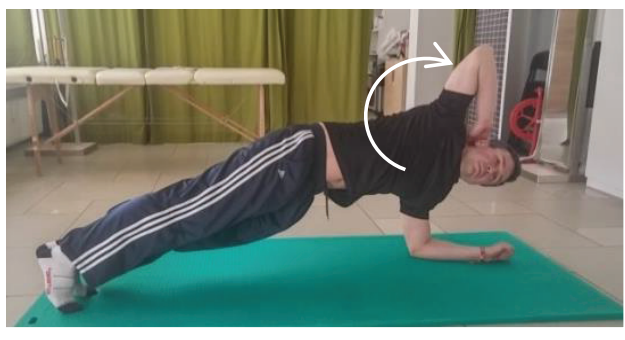

Fig.8. Plank archer turns.

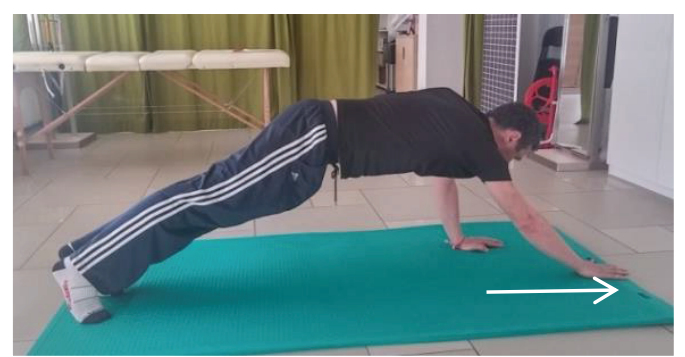

Fig. 10. Four-way reaching.

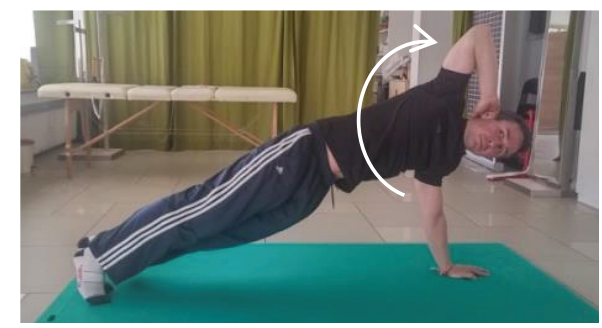

Fig. 9. Archer turns with extended elbow.

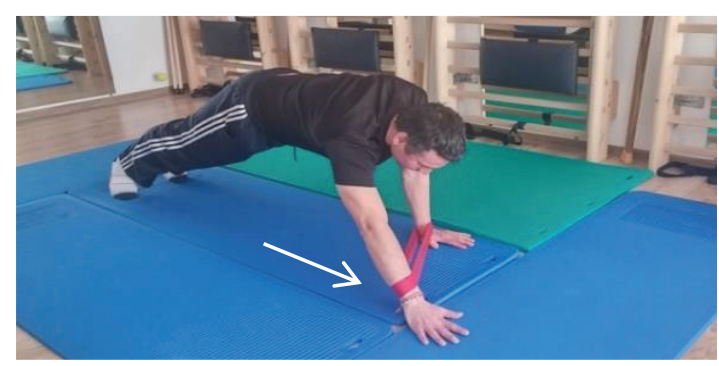

Fig. 11. Four-way reaching with Theraband loop. 
Results. The results of this study indicated that closed kinetic chain exercises, applied to our patients with SIS, significantly decreased pain, improved active pain-free shoulder ROM and scapular stability and symmetry in motion.

Pain. The data of the examination of the pain intensity showed, that at the beginning of the treatment course pain level was average 4.7 (VAS) and at the end VAS score decreased to 1.3 ( $\mathrm{P} \otimes 0,001$ ). The effect of the exercise therapy in reducing pain is mostly related with improved scapular and shoulder stability, which provides better stabilization of the humeral head and reduces compression of the subacromial soft tissues. For better results exercise therapy could be combined with other treatments, such as manual-mobilization techniques, stretching and modalities.

ROM. The findings of our study demonstrated statistically significant improvement in pain-free active shoulder ROM. At the end of the treatment course active shoulder elevation increased by $52.7^{\circ}$ for flexion and $44.8^{\circ}$ for abduction, and reached average $171.1^{\circ}$ and $157.3^{\circ}$ respectively. Improvement in shoulder ROM is mainly connected with decreasing patients' level of pain. Another reason may be improved scapular stabilization (position and motion), which is of great importance for all glenohumeral joint movements. Our findings agree with those of other researchers who have documented improvements in ROM following an exercise program in patients with SIS $(1,6,12,13)$.

Scapular stability and motion. The results revealed considerable improvement in scapular symmetry in resting position and kinematics during arm movements after the exercise therapy. Greater improvement was detected for scapular position - at the end of the treatment course there were no signs of winging or asymmetry in position of the scapula in rest or in $90^{\circ}$ shoulder abduction in most of the patients. Regardless of the significant changes in scapular stabilization full recovery of the scapular kinematics during shoulder functional movements might require a long term practice.

Conclusion. Physiotherapy for athletes with SIS is a complex process that requires detailed and comprehensive evaluation and identification of causes of impingement. In patients with impaired dynamic stabilization of the shoulder and with scapular dyskinesis exercises for muscle strength and balance and timing properties of couple forces of muscles are of particular importance.

The study supports that closed kinetic chain exercises are effective in the treatment of patients with SIS. Training of the dynamic stabilization of the shoulder and scapula contributes for the reduction of pain, improved scapular stability and symmetry, and increased pain-free ROM. When the dynamic stabilization of the shoulder and scapula is restored functional kinetic chain exercises with functional muscle patterns should be included in the exercise treatment of SIS.

\section{Bibliography}

Azar Moezy, Saeed Sepehrifar, Masoud Solaymani Dodaran. The effects of scapular stabilization based exercise therapy on pain, posture, flexibility and shoulder mobility in patients with shoulder impingement syndrome: a controlled randomized clinical trial. Med J Islam Repub Iran. 2014; 28: 87.

Cools AM, Witvrouw EE, Declercq GA, et al. Scapular muscle recruitment patterns: trapezius muscle latency with and without impingement symptoms. Am J Sports Med 2003;31:542-9.

Dustin H. Hardwick, Justin A. Beebe, Mary Kate McDonnell, Catherine E. Lang, A Comparison of Serratus Anterior Muscle Activation During a Wall Slide Exercise and Other Traditional Exercises J Orthop Sports Phys Ther 2006;36(12):903-910.

Graichen H, Stammberger T, Bonel H, Englmeier K-H, Reiser M, Eckstein F. Glenohumeral translation during active and passive elevation of the shoulder - a 3D openMRI study. J Biomech. 2000;33(5):609-13.

Han KJ, Cho JH, Han SH, Hyun HS, Lee DH. Subacromial impingement syndrome secondary to scapulothoracic dyskinesia. Knee Surg Sport Tr A. 2012 Oct;20(10):1958-60.

Kuhn JE. Exercise in the treatment of rotator cuff impingement: a systematic review and a synthesized evidence-based rehabilitation protocol. J Shoulder Elb Surg. 2009;18(1):138-60.

Laudner KG, Myers JB, Pasquale MR, et al. Scapular dysfunction in throwers with pathologic internal impingement. J Orthop Sports Phys Ther 2006;36:485-94.

Ludewig P, Hoff M, Osowski E, Meschke S, Rundquist P. Relative balance of serratus anterior and upper trapezius muscle activity during push-up exercises. Am J Sports Med. 2004;32:484-493.

Ludewig PM, Cook TM. Alterations in shoulder kinematics and associated muscle activity in people with symptoms of shoulder impingement. Phys Ther. 2000;80(3):276-91.

Ludewig PM, Reynolds JF. The association of scapular kinematics and glenohumeral joint pathologies. J Orthop Sports Phys Ther 2009;39:90-104. 
Lukasiewicz A, McClure P, Michener L, Pratt N, Sennett B. Comparison of 3-dimensional scapular position and orientation between subjects with and without shoulder impingement. J Orthop Sports Phys. 1999;29(10):574. McClure PW, Bialker J, Neff N, Williams G, Karduna A. Shoulder function and 3-dimensional kinematics in people with shoulder impingement syndrome before and after a 6-week exercise program. Phys Ther. 2004;84(9):832-48.

Michener LA, Walsworth MK, Burnet EN. Effectiveness of rehabilitation for patients with subacromial impingement syndrome: a systematic review. J Hand Ther.
2004;17(2):152-64

Voight ML, Thomson BC. The role of the scapula in the rehabilitation of shoulder injuries. J Athl Training. 2000;35(3):364.

\section{Correspondence address:}

Assoc. Prof. Lyubomira Sazdova, PhD

NSA “Vassil Levski”, 1700 Studentski grad

Mobile: +359892299772

e-mail: 1 sazdova@abv.bg 